\title{
AN EVALUATION OF THE FACTOR STRUCTURE OF THE INSTRUMENTAL ACTIVITIES OF DAILY LIVING INVOLVEMENT AND CAPACITY SCALES OF THE MINIMUM DATA SET FOR HOME CARE FOR ELDERLY CHINESE COMMUNITY DWELLERS IN HONG KONG
}

\author{
Doris $Y \mathrm{P}$ Leung, $\mathrm{PhD}$ \\ Angela $Y$ M Leung, PhD \\ Iris Chi, DSW
}

\begin{abstract}
The objective of this study was to evaluate the factor structure of the Instrumental Activities of Daily Living (IADL) Involvement and Capacity scales of the Chinese version of the Minimum Data Set-Home Care (MDS-HC), in a sample of Chinese older adults living in Hong Kong $(n=3523)$. The results of confirmatory factor analyses supported the one-factor model for both IADL Involvement and IADL Capacity scales. Evidence indicated that both scales had good internal consistency (0.88) and were reliable and valid in assessing IADL among elderly Chinese community-dwellers.
\end{abstract}

KEYWORDS. Chinese, IADL, factor structure, MDS-HC, home care, elderly community-dwellers.

Contact information:

Please address correspondence to: Iris Chi, School of Social Work, University of Southern California, USA.

Leung, Doris Y.P., School of Nursing, The University of Hong Kong, Tel no: 852-28192641. Fax no: 85228726079. E-mail:dorisl@hkucc.hku.hk.

Leung, Angela Y. M., School of Nursing and Sau Po Centre on Ageing, The University of Hong Kong, Tel no: 852-28192630. Fax no: 852-28726079. E-mail: angleung@hku.hk.

Chi, Iris, School of Social Work, University of Southern California, Tel no: 213-8211360. Fax no: 213-7400789. E-mail: ichi@usc.edu. 


\section{INTRODUCTION}

Functioning ability is a major focus in aging studies. It is commonly conceptualized as a measure of the ability to perform personal care and self-maintenance activities. Information on daily function is used in a variety of clinical (Katz, Kabeto, \& Langa, 2000), policy (Lai et al., 2009; Leichsenring \& Alaszewski, 2004) and research contexts (Lee, Chau, Hui, Chan, \& Woo, 2009; Wang, Kane, Eberly, Virnig, \& Chang, 2009). Service providers use the information to describe the stages and severity of disabling chronic diseases and to develop individualized care plans for treatment purposes. Policy makers use the information as one of the eligibility criteria for services provided by health and social services agencies and for the planning of new social policies. Researchers frequently include the measure as an outcome in their clinical studies (Friedman, Wamsley, Liebel, Saas, \& Eggert, 2009).

Functioning ability is frequently assessed in older adults according to their performance of the basic activities of daily living (ADL) - such as eating, bathing, dressing, - and the instrumental ADL (IADL) - that includes activities such as meal preparation, shopping and medication management (Spector \& Fleishman, 1998). Compared with ADLs, IADLs include more complex and higher-order activities that require higher levels of neuropsychological organization (Lawton \& Brody, 1969; Ng, Niti, Chiam, \& Kua, 2006). Both ADL and IADL measures are important for evaluating older community-dwellers who must take care of themselves or be cared for by caregivers. 
The dimensionality of a measuring tool is another critical consideration because it directly relates to the definition of the construct and to the interpretation of scale scores (Anderson \& Gerbing, 1982). IADL measures lack clear specification of the underlying construct, and there is still debate over whether IADLs should be conceptualized as a unidimensional or two-dimensional construct (Lindeboom, Vermeulen, Holman, \& De Hann, 2003; Thomas, Rockwood, \& McDowell, 1998). In the most common twodimensional conceptualization, activities related to intermediate self-care like household chores (e.g. preparing a meal, performing ordinary housework) and transport (e.g. walking, using public transportation), are categorized as physical IADLs, while those requiring higher cognitive resources for more complex self-management (e.g. managing finances or medication, using the phone) are categorized as cognitive IADLs ( $\mathrm{Ng}$ et al., 2006; Thomas et al., 1998; Wolinsky \& Johnson, 1991). However, Vittengl, White, McGovern and Morton (2006) show that a set of eight IADL items can be expressed as a single dimension. Furthermore, other studies show that the ADL and IADL items are highly correlated and hence can be combined into a single measure of functional disability (Fleishman, Spector, \& Altman, 2002; LaPlante, 2010; Spector \& Fleishman, 1998).

The Resident Assessment Instrument (RAl) is a series of standardized comprehensive assessment tools that was developed by an international group of academics, clinicians and other health care professionals for analyzing different services, such as home care, residential care, acute care and mental health care (Carpenter, 2006; Hirdes et al., 1999; Morris et al., 1990). The Minimum Data Set for Home Care (MDS-HC) is one of the RAI assessment tools specifically developed for the 
home care setting, and is also known as the RAl-Home Care (RAI-HC) (Morris et al., 2000). The MDS-HC is typically used as a screening instrument for home care providers in assessing the multiple key domains of functioning, health, social support and service use (Hirdes, 1996). The MDS-HC provides two scales that measure IADL from two different perspectives: The IADL Involvement scale aims to measure the client's involvement level, while the IADL Capacity scale assesses the client's difficulty level in performing seven specific tasks (Kwan, Chi, Lam, Lam, \& Chou, 2000). The Hong Kong-Chinese version of the MDS-HC was validated in previous studies that showed adequate reliability and criterion validity (Chou, Chi, Leung, Wu, \& Liu, 2001; Kwan et al., 2000). A few studies have examined the factor structure of some measures in other versions of MDS (Casten, Lawton, Parmelee, \& Kleban, 1999; Morris, Fries, \& Morris, 1999), but no study thus far has addressed the IADL scales in the MDS-HC. The objective of the current study is to examine the underlying factor structures of the IADL Involvement and Capacity scales in the Chinese version of the MDS-HC through confirmatory factor analysis by using a secondary data sample of Hong Kong community-dwelling elderly people who have sought long-term care services.

\section{METHODS}

\section{SAMPLE AND PROCEDURE}


We conducted a secondary analysis of data collected from a large cohort of elderly community-dwellers applying for long-term care service in Hong Kong in 2006. The MDS-HC was mandated for use as the placement assessment tool for existing service users to determine the care needs of the elderly and match them with appropriate services in Hong Kong. With the implementation of the central waiting list for government-subsidized long-term care services, the instrument has been further mandated for use in the eligibility screening of applications for long-term care services since November 2003 (Lai, Tse, \& Lau, 2008). Older adults in Hong Kong who applied for long-term care services had to be referred from designated local government departments and non-governmental organizations which provide social care services in order to be registered on the central waiting list (Social Welfare Department, 2009). Trained assessors, professionals from various disciplines (e.g. social workers, nurses, occupational therapists and physiotherapists) who had completed a five-day training program on the use of the MDS-HC assessment tool, conducted the assessment. The assessment included direct questioning of the client and the primary family caregiver - if the client was being cared for - observation of the client in the home environment, and a review of secondary documents if they were available. In the case of dubious answers, the assessor would make a further in-depth assessment to arrive at the most accurate professional judgment. For example, the assessor would ask the client's caregiver regarding receiving medication as prescribed by physician/nurse practitioner if either the client is cognitively impaired, or the caregiver administers the medications to the client. The assessor would also check the client's medical record in case the caregiver could not clearly state the details. A total of 10,331 clients on the central waiting list completed 
the MDS-HC in 2006. The sample in the current analysis consisted of 3523 elderly persons aged 60 or older, who lived in private homes and had no prior or current home care services.

\section{INSTRUMENTAL ACTIVITIES OF DAILY LIVING INVOLVEMENT AND CAPACITY ITEMS IN THE MDS-HC2.0}

There are seven items measuring the functioning of older adults in their instrumental activities of daily living (IADL) in the MDS-HC. Each item was measured from two perspectives: a) the level of involvement and b) how difficult it is or would be for older adults to carry out the activity on their own. The IADL Involvement scale measured the client's performance at home or in the community during the seven days prior to the assessment date using a 4-point Likert scale ranging from $0=$ ='independent' to $3=$ 'totally dependent on others' when performing an activity - with one additional option when the activity did not occur at all. In line with the previous study on ADL in the MDS by Morris et al. (1999), we also converted the additional code of 'activity did not occur' to $3=$ 'totally dependent on others' in the analysis. The IADL Capacity scale assessed the extent of difficulty when clients were performing the activity solely by themselves, using a 3-point Likert scale, ranging from $0=$ 'no difficulty' to $2=$ 'great difficulty'.

DATA ANALYSIS 
Confirmatory factor analysis (CFA) was used to assess the factor structure of each of the IADL Involvement and Capacity scales. A one-factor model with all the items as indicators and a two-factor model with three items (managing finance, managing medication and phone use) corresponding to the cognitive IADL factor and four items (meal preparation, ordinary housework, shopping and transport) corresponding to the physical IADL factor were fitted to the covariance matrix of the corresponding IADL items for the Involvement and Capacity scales respectively. To test the stability of the resulting factor structure after identifying the most economical model, we performed a cross-validation examination of the model by randomly splitting the sample into half and by gender. The reliability of the two scales was assessed by computing and interpreting Cronbach's alpha. Scores of the IADL Involvement and Capacity scales were computed by summing the corresponding items. Katz et al., (2000) found, in a national survey in the United States, that women reported more IADL impairment and were more likely to be living alone than men. Hence, we compared the IADL scores by gender and their living status using the Mann-Whitney test with SPSS17.0. For each comparison, the standardized mean difference effect sizes (Cohen's $d$ ) were computed and values of 0.2 , 0.5 and 0.8 and greater were considered as small, medium and large differences respectively (Cohen, 1992). For all tests, a p-value $<0.05$ is considered as statistically significant.

All the CFAs were performed by the EQS 6.0 package (Bentler, 2006) using the maximum likelihood estimation with a robust procedure to adjust for non-multivariate normality of the data (Satorra \& Bentler, 1994). Assessment of the model's fit to the data was based on four fit indices: (a) Robust Comparative Fit Index (R-CFI); Bentler 
1990), (b) Robust Normed Fit Index (R-NFI; Benlter \& Bonnett, 1980), (c) standardized root mean squared residuals (standardized root mean squared residuals (SRMR); Bentler, 2006), and (d) root mean square error of approximation (RMSEA; Browne \& Cudeck, 1993). The cutting values of $\mathrm{R}-\mathrm{CFI}>0.90, \mathrm{R}-\mathrm{NFI}>0.90, \mathrm{SRMR}<0.08$, and RMSEA $<0.08$ indicate a good fit to the data and will not be rejected (Hoyle, 1995). We also reported the robust $x^{2}\left(R-x^{2}\right)$ and its associated degree of freedom ( $d f$ for completeness, although they were not used for model evaluation because the $R-x^{2}$ test is sensitive to sample size (Hu, Bentler, \& Kano, 1992).

\section{RESULTS}

\section{CHARACTERISTICS OF THE SUBJECTS}

The average age of the subjects was 79.6 years $(S D=7.5)$. Sixty percent were females $(n=2122)$ and $21 \%$ were living alone $(n=736)$. Most had no formal education $(57 \%$, $n=2008)$ or a minimum primary education $(32 \%, n=1125): 6 \%$ had completed primary education ( $n=217)$ and only $5 \%$ had at least some secondary education $(n=173)$. Fortythree percent were married ( $n=1496), 50 \%$ were widowed $(n=1743), 5 \%$ had never married ( $n=163)$, and 3\% were divorced/separated/cohabiting ( $n=121)$.

Table 1 presents frequencies and mean scores of the individual IADL items, with a higher mean Involvement score indicating higher dependency on others and a higher Capacity score indicating more difficulty performing tasks. The respondents were least 
frequently dependent in the case of the cognitive IADL items concerning phone use and managing medication and to a lesser extent in managing finances on the Involvement scale. A similar pattern was observed regarding their level of difficulty in performing the IADLs on the Capacity scale.

\section{FACTOR STRUCTURE OF THE INSTRUMENTAL ACTIVITIES OF DAILY LIVING INVOLVEMENT SCALE}

Examination of the fit indices in Table 2 reveals that, although the Root Mean Square Error of Approximation (RMSEA) values exceeded the cut-off of 0.08 , both the one-factor and two-factor models provided good fits for the data of the IADL Involvement scale. This was supported by the other indices (R-CFI; R-NFI and SRMR), for the overall sample, males and females, and both split halves. The CFA results further suggested that the two-factor model provided a better fit than the one-factor model. However, a closer examination of the factor correlations between the physical and cognitive IADL factors in the two-factor model showed that they were highly correlated $(>0.88)$, using Cohen's criterion (Cohen, 1988) in the overall sample and gender and random split subsamples, which indicates they are combinable into a single measure (Spector \& Fleishman, 1998). Table 2 also shows that all the standardized factor loadings of the one-factor model for the IADL Involvement scale were greater than 0.50 for the overall sample as well as the split halves and male and female sub-samples. In addition, the Cronbach alpha for the overall sample was 0.882 , which was greater than the cut-off point of 0.7 (Kline, 2000). Therefore, we could conclude that the IADL Involvement scale was reliable in the sample of the Hong Kong community-dwelling elderly. 


\section{FACTOR STRUCTURE OF INSTRUMENTAL ACTIVITIES OF DAILY LIVING CAPACITY SCALE}

Table 3 shows the two-factor model provides a superior fit to the one-factor model for the data of the IADL Capacity scale for the overall sample and the gender and the split half sub-samples. However, the high factor correlations between the physical and cognitive IADL factors $(>0.75)$ indicate that the two factors are highly correlated. The one-factor model gave marginally acceptable fits except for the female sub-sample, where a good fit was observed but it was primarily chosen for its efficiency (Spector \& Fleishman, 1998). Again, the values of RMSEA were greater than the cut-off of 0.08 for the overall samples and all the sub-samples. Table 3 shows that all the standardized factor loadings were greater than 0.54 for the one-factor structure of the IADL Capacity scale. The Cronbach alpha value of the IADL Capacity scale was 0.881 for the overall sample, suggesting the scale has acceptable internal consistency for the sample of elderly community-dwellers.

\section{COMPARISON OF INSTRUMENTAL ACTIVITIES OF DAILY LIVING INVOLVEMENT AND CAPACITY SCORES}

Scores of the IADL Involvement and Capacity scales were then computed by summing the corresponding items. Ranging from 0 to 21, the IADL Involvement scores indicate the clients' dependency level in performing IADL. The higher the Involvement score, the higher the client's level of dependency. Similarly, the level of capacity the client 
achieved when performing IADL was reflected by the Capacity scores, ranging from 0 to 14 , with higher scores indicating the increasing difficulty clients found when performing the activities on their own.

Moderate levels of dependency were reported in both the IADL Involvement $(M=13.2, S D=5.9$ out of $0-21)$ and Capacity $(M=9.7, S D=3.7$ out of $0-14)$ in the community-dwelling sample. The effect sizes in the IADL Involvement and Capacity scales with respect to gender were small, although females scored significantly lower than males on both the IADL Involvement scale $(M=12.8, S D=6.1$ vs. $M=13.8, S D=5.7$; $p<0.001 ; d=0.08)$ and the Capacity scale $(M=9.6, S D=3.8$ vs. $M=10.0, S D=3.5 ; p<0.001 ;$ $d=0.06)$. There were small to medium effect sizes in the two mean scores concerning living arrangement; older adults living alone had significantly lower scores on both the IADL Involvement scale $(M=8.9, S D=6.1$ vs. $M=14.4, S D=6.1 ; p<0.001 ; d=0.38)$ and the Capacity scale $(M=7.4, S D=3.8$ vs. $M=10.4, S D=3.4 ; p<0.001 ; d=0.33)$, compared to those living with others.

\section{CONCLUSION}

Measuring instrumental activities of daily living (IADL) among older adults is valuable because of its usefulness in various clinical, policy and research contexts. In our study, we examined the factor structure of the IADL Involvement and Capacity scales of the Chinese version of the MDS-HC through confirmatory factor analysis, using a large sample of community-dwelling older adults seeking long-term care services in Hong 
Kong. Although the two-factor model was a better fit than the one-factor model, the current findings support the one-factor structure of both IADL Involvement and Capacity scales - given that the physical IADL and cognitive IADL factors were highly correlated $(\geq 0.80)$, indicating the two factors were combinable. We were also able to replicate the factor structures of the two IADL scales in two random sub-samples of females and males; internal consistency estimates for the two IADL scales were satisfactory.

$\mathrm{Ng}$ et al. (2006) also found that a two-factor structure provided a better fit for an eight-item IADL Involvement scale, with a moderate correlation in their sample of Asian older adults between the physical and cognitive IADL factors (0.61). The discrepancy in the factor correlations of the two studies might be due to the differences in respondents' characteristics. Compared with respondents randomly drawn from the community, our own respondents (all applying for subsidized long-term care services) were older and frailer - many needed at least some help with performing the seven IADL tasks. Previous studies also showed that a greater proportion of older than younger adults had difficulty performing physical IADL activities, but such a pattern was not observed in cognitive IADL activities (LaPlante, 2010; Niti, Ng, Chiam, \& Kua, 2007). Further studies should investigate the heterogeneity in the seven IADL items of the MDS by age among elderly Chinese.

The results of our study have significant clinical implications for healthcare services. In response to the increasing demand for home-care support for disabled elderly people in the community, healthcare organizations in many countries, including Hong Kong, are developing programs to provide a myriad of community support services (Lai et al., 2009). The clinical assessment of daily function and disability thus 
becomes an integral part of the clinical decision-making process in service provision. The IADL Involvement scale in the Hong Kong Chinese version of MDS-HC was shown to have an acceptance factorial validity and is therefore a valid overall measure of the self-reported IADL Involvement of the community-dwelling elderly. Healthcare providers can use the overall score of the IADL Involvement scale with confidence as a reliable and essential component for the determination of a client's eligibility for service. The IADL Capacity scale, on the other hand, offers healthcare providers with more informative guidance in designing the individualized care plan that will best fit the particular needs of each elderly adult, especially those living alone who were found to be more vulnerable in their functional daily living activities.

There are a number of limitations to the current study that are worth noting. First, the one-factor solution for the IADL Involvement and the IADL Capacity scales was replicated in the same sample. In order to arrive at firm conclusions about the bestfitting factor solutions, cross-validation using new samples would be necessary. Second, given the cross-sectional data used in the current study, we were unable to examine the predictive validity of the scales. Hence, further studies utilizing a longitudinal design will be desirable for examining the predictive validity as well as stability of the factor structures of the two IADL scales over time. Third, although the sample consisted of a large cohort of older Hong Kong adults seeking long-term care services for the first time, replication of our study with more representative samples of the whole Chinese elderly community would definitely further enhance the generalizability of the study's results. 


\section{REFERENCES}

Anderson, J. C., \& Gerbing, D. W. (1982). Some methods for respecifying measurement models to obtain unidimensional construct measurement. Journal of Marketing Research, 19, 453-460.

Bentler, P. M. (1990). Comparative fit indexes in structural models. Psychological Bulletin, 107, 238-246.

Bentler, P. M. (2006). EQS structural equations program manual. Encino, CA: Multivariate Software.

Bentler, P. M., \& Bonnett, D. G. (1980). Comparative fit indices in structural models. Psychological Bulletin, 88, 588-600.

Browne, M. W., \& Cudeck, R. (1993). Alternative ways of assessing model fit. In K. A. Bollen \& J. S. Long (Eds.), Testing structural equation models (pp. 136-161). Newbury Park, CA: Sage.

Carpenter, G. I. (2006). Accuracy, validity and reliability in assessment and in evaluation of services for older people: The role of the interRAI MDS assessment system. Age and Ageing, 35(4), 327.

Casten, R., Lawton, M. P., Parmelee, P. A., \& Kleban, M. H. (1998). Psychometric characteristics of the Minimum Data Set I: Confirmatory factor analysis. Journal of the American Geriatrics Society, 46, 726-735.

Chou, K. L., Chi, I., Leung, A. C. T., Wu, Y. M., \& Liu, C. P. (2001). Validation of Minimum Data Set for nursing home in Hong Kong Chinese Elders. Clinical Gerontologist, 23(1/2), 43-54. 
Cohen, J. (1988). Statistical power analysis for the behavioural science $\left(2^{\text {nd }}\right.$ ed.). Hillsdale, NJ: Lawrence Earlbaum Associates.

Cohen, J. (1992). A power primer. Psychological Bulletin, 112, 155-159.

Fleishman, J. A., Spector, W. D., \& Altman, B. M. (2002). Impact of differential item functioning on age and gender differences in functional disability. Journal of Gerontology: Social Sciences, 57, S275-S284.

Friedman, B.,Wamsley, B. R., Liebel, D. V., Saad, Z. B., \& Eggert, G. M. (2009). Patient satisfaction, empowerment, and health and disability status effects of a disease management-health promotion nurse intervention among medicare beneficiaries with disabilities. The Gerontologist, 49(6), 778-792.

Hirdes, J. P. (1996). Commentary on the proposed common assessment instrument (CAI) for long term care services. Canada: Research Department \& Canadian Collaborating Centre.

Hirdes, J. P., Fries, B. E., Morris, J. N., Steel, K., Mor, V., Frijters, D., LaBine, S., Schalm, C., Stones, M. J., Teare, G., Smith, T., Marhaba, M., Perez, E., Jonsson, P. (1999). Integrated health information systems based on the RAI/MDS series of instruments. Healthcare management forum, 12(4), 30. Hoyle, R. (1995). The structural equation modeling approach: Basic concepts and fundamental issues. In R. H. Hoyle (Ed.), Structural equation modeling, concepts, issues, and applications (pp. 1-15). Thousand Oaks, CA: Sage Publications.

Hu, L., Bentler, P. M., \& Kano, Y. (1992). Can test statistics in covariance structure analysis be trusted? Psychological Bulletin, 112, 351-362. 
Katz, S. J., Kabeto, M., Langa, K. M. (2000). Gender disparities in the receipt of home care for elderly people with disability in the United States. Journal of American Medical Association, 284(23), 3022-3027.

Kline, P. (2000). A psychometrics primer. Free Association Books, London.

Kwan, C.W., Chi, I., Lam, T. P., Lam, K. F., \& Chou, K. L. (2000) Validation of Minimum Data Set for Home Care assessment instrument (MDS-HC) for Hong Kong Chinese elders. Clinical Gerontologist, 21(4), 35-48.

Lai, W. W. L., Chan, J. P. Y., Choi, P. K. K., Ngai, L. C. T., Mar, C. S. F., Chan, E. Y. L., Lam, P. Y. P., \& Lam, F. N. Y. (2009). Occupational therapy in the integrated elderly and community support services for the elderly in Hong Kong. Hong Kong Journal of Occupational Therapy, 19(1), 27-30.

Lai, C. K. Y., Tse, M. M. Y., \& Lau, L. K. P. (2008). Placement appropriateness for seniors into long-term care - A neglected area of research. Asian Journal of Gerontology \& Geriatrics, 3, 34-39.

LaPlante, M. P. (2010). The classic measure of disability in activities of daily living is biased by age but an expanded IADL/ADL measure is not. Journal of Gerontology: Social Sciences, 10.1093/geronb/gbp129.

Lawton, M. P., \& Brody, E. (1969). Assessment of older people: Self-maintaining and instrumental activities of daily living. The Gerontologist, 9, 179-186.

Lee, J. S. W., Chau, P. P. H., Hui, E., Chan, F., \& Woo, J. (2009). Survival prediction in nursing home residents using the minimum data set subscales: ADL selfperformance hierarchy, cognitive performance and the changes in health, end- 
stage disease and symptoms and sign scales. European Journal of Public Health, 19(3), 308-312.

Leichsenring, K., \& Alaszewski, A. M. (2004). Providing integrated health and social care for older persons: a European overview of issues at stake. Farnham: Ashgate.

Lindeboom, R., Vermeulen, M., Holman, R., \& De Hann, R. J. (2003). Activities of daily living instruments: Optimizing scales for neurologic assessments. Neurology, 60, 738-742.

Morris, J. N., Fries, B. E., \& Morris, S. A. (1999). Scaling ADLs within the MDS. Journal of Gerontology: Medical Sciences, 54(11), 546-553.

Morris, J. N., Fries, B. E., Bernabei, R., Steel, K., Ikegami, N., Carpenter, I., et al. (2000). RAI-Home Care (RAI-HC) assessment manual for version 2.0. Marblehead, MA: Opus Communications.

Morris, J. N., Hawes, C., Fries, B. E., Phillips, C. D., Mor, V., Katz, S., Murphy, K., Drugovich, M. L., \& Friedlob, A. S. (1990). Designing the national resident assessment instrument for nursing homes. Gerontologist, 30(3), 293-307.

Ng, T.-P., Niti, M., Chiam, P.-C., Kua, E.-H. (2006). Physical and cognitive domains of the instrumental activities of daily living: validation in a multiethnic population of Asian older adults. Journal of Gerontology: Medical Sciences, 61A(7), 726-735.

Niti, M., Ng, T.-P., Chiam, P.-C., \& Kua, E.-H. (2007). Item response bias was present in instrumental activity of daily living scale in Asian older adults. Journal of Clinical Epidemiology, 60, 366-374. 
Satorra, A., \& Benlter, P. M. (1994). Corrections to test statistics and standard errors in covariance structure analysis. In A. von Eye \& C. C. Clogg (Eds.), Latent variables analysis: Applications for developmental research (pp.399-419). Thousand Oaks, CA: Sage.

Social Welfare Department (2009). Central waiting list for subsidised long term care services. Retrieved on 9 Sept 2010 (http://www.swd.gov.hk/en/index/site_pubsvc/page_elderly/sub_centralwai/).

Spector, W. D., \& Fleishman, J. A. (1998). Combining activities of daily living with instrumental activities of daily living to measure functional disability. Journal of Gerontology: Series B: Psychological Sciences and Social Sciences, 53, S46S57.

Thomas, V. S., Rockwood, K., \& McDowell, I. (1998). Multidimensionality in instrumental and basic activities of daily living. Journal of Clinical Epidemiology, 51(4), 315321.

Vittengl, J. R., White, C. N., McGovern, R. J., \& Morton, B. J. (2006). Comparative validity of seven scoring systems for the instrumental activities of daily living in rural elders. Aging and Mental Health, 10, 40-47.

Wang, J., Kane, R. L., Eberly, L. E., Virnig, B. A., \& Chang, L.-H. (2009). The effects of resident and nursing home characteristics on activities of daily living. Journal of Gerontology: Medical Sciences, 64A(4), 473-480.

Wolinsky, F. D., Johnson, R. J. (1991). The use of health services by older adults. Journal of Gerontology: Social Sciences, 46, S345-S357. 
TABLE 1 Summary statistics for Instrumental Activities of Daily Living (IADL) (n = 3523)

\begin{tabular}{|c|c|c|c|c|c|}
\hline \multirow[b]{2}{*}{ IADL Involvement scale } & \multicolumn{4}{|c|}{ Percentage reporting } & \multirow[b]{2}{*}{$\begin{array}{c}\text { Mean score } \\
\text { (SD) }\end{array}$} \\
\hline & $\begin{array}{c}\text { Independent } \\
\text { (0) }\end{array}$ & $\begin{array}{c}\text { Needs some } \\
\text { help (1) }\end{array}$ & $\begin{array}{c}\text { Needs full } \\
\text { help (2) }\end{array}$ & $\begin{array}{c}\text { By others } \\
\text { (3) }\end{array}$ & \\
\hline Meal preparation & 12.0 & 14.5 & 16.0 & 57.5 & $2.19(1.08)$ \\
\hline Ordinary housework & 10.7 & 17.5 & 15.5 & 56.4 & $2.18(1.06)$ \\
\hline Managing finance & 18.5 & 15.5 & 20.2 & 45.8 & $1.93(1.16)$ \\
\hline Managing medication & 32.9 & 25.9 & 26.3 & 14.9 & $1.23(1.06)$ \\
\hline Phone use & 44.9 & 14.5 & 8.0 & 32.5 & $1.28(1.32)$ \\
\hline Shopping & 9.7 & 16.3 & 21.5 & 52.5 & $2.17(1.02)$ \\
\hline Transport & 8.6 & 14.6 & 22.2 & 54.6 & $2.23(0.99)$ \\
\hline \multicolumn{6}{|c|}{ Percentage reporting } \\
\hline IADL Capacity scale & $\begin{array}{c}\text { No difficulty } \\
(0)\end{array}$ & $\begin{array}{c}\text { Some } \\
\text { difficulty (1) }\end{array}$ & $\begin{array}{c}\text { Great } \\
\text { difficulty (2) }\end{array}$ & & $\begin{array}{c}\text { Mean score } \\
\text { (SD) }\end{array}$ \\
\hline Meal preparation & 6.9 & 24.2 & 68.9 & & $1.62(0.61)$ \\
\hline Ordinary housework & 5.9 & 26.7 & 67.4 & & $1.62(0.59)$ \\
\hline Managing finance & 17.4 & 27.6 & 55.0 & & $1.38(0.76)$ \\
\hline Managing medication & 28.9 & 39.6 & 31.5 & & $1.03(0.78)$ \\
\hline Phone use & 37.8 & 32.0 & 30.2 & & $0.92(0.82)$ \\
\hline Shopping & 6.1 & 26.0 & 67.9 & & $1.62(0.60)$ \\
\hline Transport & 6.2 & 32.2 & 61.6 & & $1.55(0.61)$ \\
\hline
\end{tabular}

Response categories for the IADL Involvement scale: 0 = 'Performed independently', 1 = 'Performed with help some of the time', 2 = 'Performed with help all the time', 3 = 'Performed by others', and $8=$ 'Activity did not occur' during the last 7 days. Category 8 combined with category 3; Response categories for the IADL Capacity scale: 0 = 'Performed with no difficulty', 1 = 'Performed with some difficulty', and 2 = 'Performed with great difficulty' during the last 7 days; SD = Standard deviation. 
TABLE 2 Results of confirmatory factor analysis of the IADL Involvement Scale for the total sample, random and gender sub-samples

\begin{tabular}{|c|c|c|c|c|c|}
\hline Fit statistics & $\begin{array}{c}\text { Total } \\
(\mathrm{n}=3523)\end{array}$ & $\begin{array}{c}\text { Male } \\
(\mathrm{n}=1401)\end{array}$ & $\begin{array}{c}\text { Female } \\
(\mathrm{n}=2122)\end{array}$ & $\begin{array}{l}\text { Split half } 1 \\
(\mathrm{n}=1761)\end{array}$ & $\begin{array}{l}\text { Split half } 2 \\
(\mathrm{n}=1762)\end{array}$ \\
\hline \multicolumn{6}{|c|}{ One-factor model $(d f=14)$} \\
\hline $\mathrm{R}-\chi^{2}$ & 758.03 & 298.33 & 450.18 & 439.11 & 334.53 \\
\hline R-NFI & 0.936 & 0.922 & 0.940 & 0.930 & 0.940 \\
\hline R-CFI & 0.937 & 0.925 & 0.946 & 0.932 & 0.942 \\
\hline R-RMSEA & 0.123 & 0.120 & 0.121 & 0.131 & 0.114 \\
\hline SRMR & 0.050 & 0.053 & 0.049 & 0.050 & 0.050 \\
\hline \multicolumn{6}{|c|}{ Two-factor model $(d f=13)$} \\
\hline $\mathrm{R}-\chi^{2}$ & 573.01 & 223.75 & 343.02 & 323.53 & 263.07 \\
\hline R-NFI & 0.951 & 0.941 & 0.957 & 0.948 & 0.953 \\
\hline R-CFI & 0.952 & 0.944 & 0.959 & 0.950 & 0.955 \\
\hline R-RMSEA & 0.111 & 0.108 & 0.109 & 0.117 & 0.105 \\
\hline SRMR & 0.042 & 0.044 & 0.041 & 0.043 & 0.043 \\
\hline \multicolumn{6}{|c|}{ Standardized solution of the one-factor model } \\
\hline Item & $\mathrm{F} 1$ & $\mathrm{~F} 1$ & $\mathrm{~F} 1$ & $\mathrm{~F} 1$ & $\mathrm{~F} 1$ \\
\hline Meal preparation & 0.835 & 0.794 & 0.854 & 0.839 & 0.830 \\
\hline Ordinary housework & 0.856 & 0.840 & 0.864 & 0.853 & 0.859 \\
\hline Managing finance & 0.811 & 0.804 & 0.820 & 0.804 & 0.819 \\
\hline Managing medication & 0.566 & 0.541 & 0.580 & 0.574 & 0.558 \\
\hline Phone use & 0.597 & 0.544 & 0.627 & 0.609 & 0.584 \\
\hline
\end{tabular}




\begin{tabular}{|c|c|c|c|c|c|c|c|c|c|c|}
\hline Shopping & \multicolumn{2}{|c|}{0.812} & \multicolumn{2}{|c|}{0.827} & \multicolumn{2}{|c|}{0.807} & \multicolumn{2}{|c|}{0.818} & \multicolumn{2}{|c|}{0.804} \\
\hline Transport & \multicolumn{2}{|c|}{0.562} & \multicolumn{2}{|c|}{0.597} & \multicolumn{2}{|c|}{0.544} & \multicolumn{2}{|c|}{0.564} & \multicolumn{2}{|c|}{0.560} \\
\hline \multicolumn{11}{|c|}{ Standardized solution of the two-factor model } \\
\hline Item & F1 & F2 & F1 & F2 & F1 & F2 & F1 & F2 & F1 & F2 \\
\hline Meal preparation & 0.853 & - & 0.812 & - & 0.870 & - & 0.863 & - & 0.844 & - \\
\hline Ordinary housework & 0.876 & - & 0.864 & - & 0.880 & - & 0.879 & - & 0.874 & - \\
\hline Managing finance & - & 0.858 & - & 0.862 & - & 0.862 & - & 0.856 & - & 0.862 \\
\hline Managing medication & - & 0.618 & - & 0.593 & - & 0.629 & - & 0.631 & - & 0.604 \\
\hline Phone use & - & 0.639 & - & 0.586 & - & 0.667 & - & 0.658 & - & 0.619 \\
\hline Shopping & 0.803 & - & 0.820 & - & 0.801 & - & 0.803 & - & 0.800 & - \\
\hline Transport & 0.555 & - & 0.590 & - & 0.539 & - & 0.554 & - & 0.556 & - \\
\hline Factor correlation & \multicolumn{2}{|c|}{0.895} & \multicolumn{2}{|c|}{0.884} & \multicolumn{2}{|c|}{0.906} & \multicolumn{2}{|c|}{0.881} & \multicolumn{2}{|c|}{0.900} \\
\hline
\end{tabular}


TABLE 3 Results of confirmatory factor analysis of the IADL Capacity scale for the total sample, random and gender sub-samples

\begin{tabular}{|c|c|c|c|c|c|}
\hline Fit statistics & $\begin{array}{c}\text { Total } \\
(\mathrm{n}=3523)\end{array}$ & $\begin{array}{c}\text { Male } \\
(\mathrm{n}=1401)\end{array}$ & $\begin{array}{c}\text { Female } \\
(\mathrm{n}=2122)\end{array}$ & $\begin{array}{l}\text { Split half } 1 \\
(\mathrm{n}=1761)\end{array}$ & $\begin{array}{l}\text { Split half } 2 \\
(n=1762)\end{array}$ \\
\hline \multicolumn{6}{|c|}{ One-factor model $(d f=14)$} \\
\hline $\mathrm{R}-\chi^{2}$ & 1119.38 & 516.30 & 586.75 & 567.37 & 563.17 \\
\hline R-NFI & 0.890 & 0.850 & 0.913 & 0.893 & 0.884 \\
\hline R-CFI & 0.891 & 0.853 & 0.915 & 0.895 & 0.887 \\
\hline R-RMSEA & 0.150 & 0.160 & 0.139 & 0.150 & 0.149 \\
\hline SRMR & 0.071 & 0.080 & 0.066 & 0.070 & 0.073 \\
\hline \multicolumn{6}{|c|}{ Two-factor model $(d f=13)$} \\
\hline $\mathrm{R}-\chi^{2}$ & 577.01 & 274.52 & 291.18 & 312.24 & 274.30 \\
\hline R-NFI & 0.943 & 0.920 & 0.957 & 0.941 & 0.944 \\
\hline $\mathrm{R}-\mathrm{CFI}$ & 0.944 & 0.924 & 0.959 & 0.943 & 0.946 \\
\hline R-RMSEA & 0.111 & 0.120 & 0.100 & 0.114 & 0.107 \\
\hline SRMR & 0.046 & 0.054 & 0.042 & 0.048 & 0.046 \\
\hline \multicolumn{6}{|c|}{ Standardized solution of the one-factor model } \\
\hline Item & $\mathrm{F} 1$ & $\mathrm{~F} 1$ & F1 & $\mathrm{F} 1$ & $\mathrm{~F} 1$ \\
\hline Meal preparation & 0.832 & 0.787 & 0.855 & 0.825 & 0.838 \\
\hline Ordinary housework & 0.847 & 0.809 & 0.865 & 0.834 & 0.860 \\
\hline Managing finance & 0.752 & 0.733 & 0.768 & 0.757 & 0.747 \\
\hline Managing medication & 0.561 & 0.550 & 0.569 & 0.579 & 0.543 \\
\hline Phone use & 0.556 & 0.536 & 0.569 & 0.566 & 0.545 \\
\hline
\end{tabular}




\begin{tabular}{|c|c|c|c|c|c|c|c|c|c|c|}
\hline Shopping & \multicolumn{2}{|c|}{0.822} & \multicolumn{2}{|c|}{0.823} & \multicolumn{2}{|c|}{0.829} & \multicolumn{2}{|c|}{0.818} & \multicolumn{2}{|c|}{0.827} \\
\hline Transport & \multicolumn{2}{|c|}{0.706} & \multicolumn{2}{|c|}{0.725} & \multicolumn{2}{|c|}{0.698} & \multicolumn{2}{|c|}{0.716} & \multicolumn{2}{|c|}{0.697} \\
\hline \multicolumn{11}{|c|}{ Standardized solution of the two-factor model } \\
\hline Item & $\mathrm{F} 1$ & F2 & F1 & F2 & $\mathrm{F} 1$ & F2 & $\mathrm{F} 1$ & F2 & F1 & F2 \\
\hline Meal preparation & 0.852 & - & 0.820 & - & 0.866 & - & 0.847 & - & 0.856 & - \\
\hline Ordinary housework & 0.873 & - & 0.845 & - & 0.882 & - & 0.861 & - & 0.883 & - \\
\hline Managing finance & - & 0.834 & - & 0.809 & - & 0.852 & - & 0.830 & - & 0.839 \\
\hline Managing medication & - & 0.686 & - & 0.701 & - & 0.676 & - & 0.693 & - & 0.681 \\
\hline Phone use & - & 0.677 & - & 0.681 & - & 0.675 & - & 0.684 & - & 0.671 \\
\hline Shopping & 0.818 & - & 0.811 & - & 0.832 & - & 0.815 & - & 0.822 & - \\
\hline Transport & 0.697 & - & 0.715 & - & 0.692 & - & 0.706 & - & 0.689 & - \\
\hline Factor correlation & 0.7 & & & & & & & & & 9 \\
\hline
\end{tabular}

R- $\chi^{2}=$ Robust chi-square statistic; R-NFI = Robust Normed Fit Index; R-CFI = Robust Comparative Fit Index; R-RMSEA = Robust Root Mean Square Error of Approximation, SRMR $=$ Standardized Root Mean Squared Residuals 